\title{
Investigating the Influence of Sound Design for Inducing Anxiety in Virtual Public Speaking
}

\author{
Enora Gabory \\ IMT Atlantique \\ Nantes, France \\ enora.gabory@gmail.com
}

\author{
Mathieu Chollet \\ IMT Atlantique \\ Nantes, France \\ mathieu.chollet@imt-atlantique.fr
}

\begin{abstract}
Virtual reality has demonstrated successful outcomes for treating social anxiety disorders, or helping to improve social skills. Some studies showed that various factors can impact the level of participants' anxiety during public speaking. However, the influence of sound design on this anxiety has been less investigated, and it is necessary to study the possible impacts that it can have. In this paper, we propose a model relating sound design concepts to presence and anxiety during virtual reality interactions, and present a protocol of a future experimental study aimed at investigating how sound design and in particular sound distractions can influence anxiety during public speaking simulations in virtual environments.
\end{abstract}

\section{CCS CONCEPTS}

- Human-centered computing $\rightarrow$ HCI design and evaluation methods.

\section{KEYWORDS}

public speaking anxiety, virtual reality, sound design, distraction

\section{ACM Reference Format:}

Enora Gabory and Mathieu Chollet. 2020. Investigating the Influence of Sound Design for Inducing Anxiety in Virtual Public Speaking. In Companion Publication of the 2020 International Conference on Multimodal Interaction (ICMI '20 Companion), October 25-29, 2020, Virtual event, Netherlands. ACM, New York, NY, USA, 5 pages. https://doi.org/10.1145/3395035.3425227

\section{INTRODUCTION}

Social anxiety can be a debilitating problem for many people in their everyday lives, in a society where social interactions are extremely frequent in a wide variety of contexts. In particular, public speaking is a very common situation both in our personal - such as giving a speech at a birthday - and professional lives - such as giving a presentation to clients. For lots of people, this exercise can be really stressful and may even constitute a consequence of a social anxiety disorder [21]. In these cases, psychotherapy has proven to be a viable option to reduce the stress of public speaking tasks, and approaches such as Cognitive Behavioral Therapy (CBT) are potential treatments with demonstrated efficacy [17]. In

Permission to make digital or hard copies of all or part of this work for personal or classroom use is granted without fee provided that copies are not made or distributed for profit or commercial advantage and that copies bear this notice and the full citation on the first page. Copyrights for components of this work owned by others than ACM must be honored. Abstracting with credit is permitted. To copy otherwise, or republish, to post on servers or to redistribute to lists, requires prior specific permission and/or a fee. Request permissions from permissions@acm.org.

ICMI '20 Companion, October 25-29, 2020, Virtual event, Netherlands

(C) 2020 Association for Computing Machinery.

ACM ISBN 978-1-4503-8002-7/20/10 . \$ \$15.00

https://doi.org/10.1145/3395035.3425227
CBT, a particularly useful tool in the treatment of anxiety is the exposure therapy (ET) (i.e. expose participants to stimuli to trigger their anxiety), used for several types of phobias, including public speaking anxiety [17]. With the technological developments that have occurred in recent years in the field of virtual environments and virtual reality, developing virtual reality systems for ET - VRET systems - has become feasible [22]; commercial solutions have been created and a large amount of research has been devoted to assess their efficacy in comparison with traditional ET methods [18]. In the case of public speaking, whether it's for social anxiety exposure therapy, or simply for training communication skills, virtual reality has shown to be a valuable solution. Indeed, VRET to treat specific phobias was shown to lead to similar results as in vivo exposure therapy, with both solutions seeming to have a better efficiency than traditional methods relying on imagining the anxiety-provoking situations $[13,18]$. Vanni et al. showed the efficiency of VRET in the particular case of public speaking [26]. To better understand how to mitigate public speaking anxiety with VRET, and to create better adaptive virtual environments for therapy or for training, there is a need to explore in more detail which parameters of such VR simulations have an impact on inducing anxiety in order to fully understand virtual reality's potential for treating it [26].

Immersive experiences activate all of our senses, and while the visual element predominates and has indeed received a lot of attention in the research community [3], the auditory element of a VR experience should also be considered. Some studies in the literature have investigated the influence of sound design on realism or on the sense of presence felt in virtual reality [3,11, 23]. However, the impact of sound design on the anxiety triggered by a VR experience has not received much attention, particularly in the case of public speaking. We argue that sound design may have a large influence on the quality of a VR experience, and that therefore the possible impacts of sound design on VR tasks, including VRET, should be considered, for instance, how do unexpected sound distractions influence participants' experience and reduction in social anxiety.

In this paper, we first introduce related works in the use of VR for public speaking, and the influence of sound design on anxiety and performance. We then propose a conceptual model linking different concepts associated to the impact of sound design, and in section 4 we describe the experimental study that we will conduct to validate this model. Finally, we present future work in section 5 .

\section{STATE OF THE ART}

Public speaking anxiety isn't the only subject in which VRET is used: we can see in the literature that VRET has been studied for various phobias, such as acrophobia [8], agoraphobia [27], fear of crime [24], or social phobias [22]. 


\subsection{Public Speaking in Virtual Reality}

Early virtual reality public speaking simulations proposed to use virtual audiences in order to alleviate public speaking fears. North $e t$ $a l$. showed the efficiency of using virtual audiences to reduce public speaking anxiety [16], and these results were confirmed by Harris et al. [5]. A study investigated the influence on anxiety of three different types of virtual audiences: negative, neutral and positive audiences. The authors showed that in three types of audiences conditions, participants felt a significant amount of anxiety [20].

More recently, some studies explored which parameters of virtual audiences impact the level of anxiety. Mostarejan et al., investigated the effects of the number of audience members. They showed that a size of three audience members, in comparison with audience of six and fifteen members, induced the highest increase in heart rate measures, which they linked to higher levels of anxiety [14] Another study by Biesman et al. investigated the effects of ingroup bias on the degree of induced anxiety. They didn't find differences in anxiety when comparing audiences designed to be perceived as ingroup or outgroup by the subjects. However, they showed that in the ingroup condition resulted to higher feelings of presence compared to the outgroup condition. Additionally, a higher level of emotional intelligence - the ability to recognize and understand emotions - predicted a lower level in public speaking anxiety in the outgroup condition, while initial predispositions to public speaking anxiety predicted the state of anxiety in the ingroup condition [1] In a related topic of job interview simulations, Kwon et al. showed that better graphical realism, and the use of HMD or LCD screen over laptops, increased the degree of perceived anxiety [10].

When considering which parameters impact the level of anxiety, an important factor in the uses of virtual environments is the feeling of presence, defined as the perceptual illusion of "being there" [12]. This is particularly relevant for VRET, as it is assumed that high feels of presence would help to induce emotions or anxiety [9]. Lee divides presence in three psychological states: physical, i.e. experience virtual objects as actual physical objects, social, i.e. experience virtual social actors as actual social actors, and self presence, i.e. experience virtual self as the actual self [12]. Cummings and Bailenson present in a meta-analysis the effect size of various parameters on the feeling of spatial presence [3]. Tracking level, stereoscopy and field of view have a larger effect on user presence, in comparison with others features such as the presence of sounds or the image quality. In our case, because public speaking is first and foremost a social interaction, the presence sub-concept of social presence is arguably the most important to consider. We can link this to results obtained by Kishimoto and Ding [7], who studied the influences of ambiguous and negative virtual social feedback on anxiety and presence. If no significant differences where found on feels of presence, participants with social anxiety disorders presented higher level of anxiety during public speaking in front of an audience displaying ambiguous feedback compared to an audience with negative feedback.

\subsection{The Influence of Sound on Anxiety and Performance}

As seen above, the feeling of presence influences the amount of anxiety felt by users. Cummings et al. showed that using sound in a virtual environment has a medium-sized effect on presence [3]. Serafin present two types of sound design: ambient sounds, which are background sounds, and sound events, which can be predictable or not, and used for triggering users. They showed that through the sole use of sound design to represent a panoramic viewpoint, with both types of sounds and without any visual information, it is possible to create a feeling of presence [23]. In parallel, Larsson et al. showed that consistency in sound design is important: indeed, an inconsistent sound design, i.e. which is not realistic or seems unrelated to the environment, negatively impacts the feeling of presence. For example, sounds that do not match with the visual environment, such as sounds temporally desynchronized from their source, are unrealistic and reduce presence [11].

In the field of virtual environments, video games are a valuable field of study; there, sound design represents a crucial part on how emotions can be induced in the player. Toprac and Abdel-Meguid studied the impact of sound, in survival horror games, on feelings of fear, anxiety and suspense. They observed that medium volume sound effects were the most effective way to induce anxiety in games [25].

On the impact of sound on performance, some studies showed that noise distractions, such as white noise, emitted during doubletasks, which require a high level of attention, negatively impact the performance on the subsidiary task, and with no impacts on the primary task. More specifically, unpredictable distractions impact the performance more than predictable noise distractions [4]. Equivalent results are observed on subjects' performance on a complex task also requiring a high level of attention [15]. The effects of sound distractions on subsidiary task or complex tasks were also investigated by Hong. While attending to a primary task, participants had to respond to a sound distraction as a subsidiary task. Hong showed that performance on the primary task declined when participants where confronted to sound distractions [6].

We can see that sounds have different impacts, particularly on the feeling of presence as well as on the performance on complex tasks. However, it seems that the influence of sound design on participant anxiety in virtual environments hasn't really been explored yet, particularly during public speaking task. Based on the existing research presented above, we propose a model about the influence of sound design on presence and anxiety during public speaking in virtual reality.

\section{CONCEPTUAL MODEL}

The main idea behind this model is to map out how various factors of a virtual experience's sound design combine in a way that ends up impacting the level of anxiety. Based on the literature review presented in the previous section, we have outlined this model in Figure ??. In this figure, the relationships between different subjective factors and virtual reality parameters are outlined by arrows: the majority of these links have been investigated in previous studies (although in different contexts than public speaking simulations) and are represented by bold arrows. In contrast, the dotted line between auditory distractions and anxiety corresponds to an hypothesis we formulate and that we will attempt to verify in an experiment (see 4.1). 
Because of the impact of distractions on performance when tasks require a lot of attention - and we assume here it is the case of public speaking tasks, where we need to know what to say, remember the order of the presentation, speak and adopt appropriate nonverbal behaviors, all the while adapting to the audience and to the environment - we suppose that distractions will have a negative impact not just on performance but also on anxiety, both being potentially linked (e.g. a loss of performance might trigger anxiety).

\section{EXPERIMENTAL STUDY}

In order to validate the above model, we are planning to run an experimental study where we will explore the impact of sound design and in particular sound distractions on the level of anxiety and on the level of presence during public speaking in virtual reality. To that end, we will immerse participants in a virtual reality environment, consisting of a virtual amphitheater populated by a virtual audience, and containing different sound distractions. In the following subsections, we present our research hypotheses, the virtual environment we use and its visual and auditory stimuli, our experimental protocol, and the measures we plan to obtain.

Participants will be recruited among students at our institution, at a particular point in their curriculum where they have to prepare an oral presentation on an internship done during the summer. Participants will be requested to perform a public speaking presentation in front of a virtual audience provides through a HTC Vive Pro Eye head-mounted display (HMD) with an integrated audio headset and eye-tracking system. Different recordings will be obtained, using a variety of sensors: a camera and microphone will be placed in front of the participants to obtain a general view of their performance. Eye-tracking data will be collected using the HMD integrated system, and an Empatica E4 wristband will be used to record heart rate and electrodermal activity.

Our study will follow a mixed experimental design: each participant will have their presentation divided in three parts, corresponding to three experimental conditions of sound distractions, selected in a random order. Additionally, participants will be randomly assigned between two groups, corresponding to one of the two conditions of realistic or unrealistic sound design, the second one obtained by a desynchronization of sounds with visual cues. Also, in preparation of the experiment, a pilot study will be carried out in order to validate the realism of the sound environment.

\subsection{Hypotheses}

We want to see if sound design, and specifically sounds distractions, are a potential factor influencing the level of anxiety and presence during a public speaking task in virtual reality. The future experiment will try to validate these three hypotheses:

H1 A continuous sound distraction increases the level of anxiety at the begin of the diffusion of the sound. This level of anxiety decreases after a while due to an habituation effect.

H2 Random variations of the volume of a sound creates an increase in the level of anxiety.

H3 An unrealistic sound design negatively impacts the feeling of presence.

H3b An unrealistic sound design impacts the level of anxiety.
In the case of $\mathbf{H} \mathbf{3} \mathbf{b}$, we envision two possibilities of the impact that an unrealistic sound design can have on anxiety. The first possible impact is that an unrealistic sound design, by impacting negatively the feeling of presence, will reduce participants' involvement and therefore reduce anxiety. Secondly, because unrealistic sounds might be uncomfortable or disturbing, it might directly increase anxiety. It is difficult to predict which of these two impacts will happen, and if so, whether one effect will dominate.

These hypotheses will confirm the validity of our model specifically in the context of public speaking. We will measure the level of anxiety with physiological and self-reported measurements, and presence with self-reported measurements.

\subsection{Virtual environment and stimuli}

We use in this work the virtual environment (VE) Cicero, developed using the VHToolkit framework based on the Unity 3D engine [2]. This system was used in a series of studies realised at the USC Institute for Creative Technologies. Cicero contains a virtual amphitheater where a virtual audience can be spawned and configured with various parameters. In particular, the audience can be configured to display a global attitude towards the speaker by varying arousal and valence parameters. The parameters then impact nonverbal behaviors displayed by the audience members. To create this audience behavior model which generates behaviors from various levels of valence and arousal, a crowdsourcing platform was used, were participants had to select behaviors, i.e. head movements, gaze patterns, facial expressions and postures, corresponding to a particular level of valence and arousal, varying between very low, low, neutral, high and very high [2]. In our study, we will set arousal and valence parameters to fixed values in order for the audience behaviors to stay stable during the experience so that they do not interference on the level of presence and anxiety. To have an audience which looks interested by the presentation, the level of arousal will be high. The valence will be globally neutral but distributed with different values for each audience members, in order to have a bigger variety of behaviors while avoiding the impact of an audience that would be globally perceived as too positive or too negative. We integrate four audience members in the environment, on the basis of the study of Mosterajan et al. [14], and in order to have the same number of women and men in the audience, with different ethnicities, in order to avoid potential ingroup/outgroup biases [1].

In order to conduct our experiment and test our hypotheses, we added sound design in the virtual environment. Ambient sounds, including sighs, interjections and seat creaking noises were added, and associated with different behaviors of audience members. We also chose simple background sounds, played at a low volume, which we assume should only impact the feeling of presence, not anxiety. We created two variations of these various sounds, namely an unrealistic and a realistic condition, altering the particular timing of these sounds with corresponding visual cues. On the basis of the work of Larsson et al. [11], we desynchronize these sounds from the corresponding visual informations to obtain the unrealistic condition of the experiment, and keep them synchronized for the realistic condition. Finally, we include a sound distraction in the form of a sound of a group of people chatting, spatialized in 
a side room in the VE. We consider that this sound can be particularly impactful to study subjects compared to other types of distractions (e.g. alarms, environmental sounds) because of its social dimension, which could be especially disturbing in the case of users suffering from public speaking anxiety. The volume of this group chat sound is managed in different ways depending of the experimental conditions, in order to create continuous or random distraction conditions, in opposition with a condition without any sound distraction.

\subsection{Protocol}

At the beginning of the experiment, the participant will first have to fill in a number of questionnaires, and will then be equipped with the Empatica E4 wristband and the HMD. Before the experiment begins, the participant will first discover the virtual environment in a habituation sequence, consisting of the same virtual environment but without sounds and without the virtual audience. This will allows us to obtain a reference of the initial physiological state of the participant before the public speaking task. The experiment will consist in three steps of public speaking of five minutes, with short breaks between these steps. Each step corresponds randomly to one condition of the sound distraction variable: no distraction, continuous distraction, or random distractions. Additionally, the sound is configured in a unrealistic or realistic condition depending on the group condition to which the participant is assigned. Participants will be asked to prepare a presentation of a duration around twenty minutes - to make sure to have a presentation of at least fifteen minutes - and to give the slideshow of their oral presentation, to integrate it in the VE - the experimentation comes during a period of oral exams for students in which internship presentations are mandatory; we will simply ask students to reuse those presentations. After five minutes of public speaking, we will interrupt the participants, ask them questions related to their previous condition, set the condition for the next presentation step and then continue for the next step of public speaking. These breaks will help us to separate recordings of the different conditions. Once their presentations are finished, participants will fill in another set of questionnaires in order to obtain measures of their subjective level of anxiety during the three steps, their feeling of presence, and a post-test questionnaire related to their perceptions of distractions and their subjective impression of those distractions' impact on their anxiety.

\subsection{Materials and Measurements}

To obtain physiological measurements, we will use an Empatica E4 wristband, which provides electrodermal activity (EDA) and a heart rate (HR) signal from which we can calculate heart rate variability (HRV), and the eye-tracker integrated to the HMD system.

EDA corresponds to changes in electrical properties of the skin, which reflect physiological arousal. HRV is a measurement of the variability in the time intervals between heart beats, and is related to emotional stability. Eye-tracking data indicates where the subject is looking, which will enable us to know if the participant looks at the source of the sound distraction, and when.
Concerning self-reported data, participants will have to fill at the beginning of the experiment, a demographic questionnaire, the Personal Report of Confidence as a Speaker (PRCS) [19], which allows us to get a measure of the participants' public speaking anxiety levels, and an immersive tendencies questionnaire (ITQ) [28]. Between steps of the experiment, they will have to fill the PRCS in order to have intermediate measures of their anxiety. Finally, at the end of the experiments, they will have to fill a presence questionnaire (PQ) [28], and a post-test questionnaire to have measurements of their subjective perception of sound distraction and the impact of it on their anxiety.

We will use also a camera and microphone, in order to realise an analysis of the participant' non-verbal behavior, in particular postures, gestures, and vocal behavior.

\section{FUTURE WORK}

The experiment we have presented is still in preparation, and therefore our most important task is to finalise the study preparation and subsequently to recruit participants to run it. Once the study has been run and data has been collected, our next step will consist in realising statistical analyses, testing for differences between the various presented conditions in terms of physiological features and subjective presence and subjective anxiety scores, in order to validate our hypotheses.

This study's main goal is exploratory, as sound design in VRET is still a largely unstudied field. We intend to develop further our model of sound design parameters influences on the level of presence and anxiety during simulated public speaking, with the end goal of improving simulations and hopefully social anxiety treatment or social skills training outcomes. While this study focuses on sound distractions, a next step can be to improve the sound design by considering elements such as the signal-to-noise ratio, the acoustic properties of the room and other sound properties. A potential follow-up study would be more devoted to studying the influence on sounds conveying social cues, such as backchannels or disagreement sounds, and how those can be linked with audience non-verbal behaviors.

\section{ACKNOWLEDGMENTS}

This material is based upon work supported by the RFI Atlanstic 2020.

\section{REFERENCES}

[1] Lotte EJ Biesmans, Pleun JM van Hees, Lisa E Rombout, Maryam Alimardani, and Eriko Fukuda. 2020. The Effects of Ingroup Bias on Public Speaking Anxiety in Virtual Reality.. In VISIGRAPP (2: HUCAPP). 17-24.

[2] Mathieu Chollet, Nithin Chandrashekhar, Ari Shapiro, Louis-Philippe Morency, and Stefan Scherer. 2016. Manipulating the perception of virtual audiences using crowdsourced behaviors. In International Conference on Intelligent Virtual Agents. Springer, 164-174.

[3] James J Cummings and Jeremy N Bailenson. 2016. How immersive is enough? A meta-analysis of the effect of immersive technology on user presence. Media Psychology 19, 2 (2016), 272-309.

[4] Jay M Finkelman and David C Glass. 1970. Reappraisal of the relationship between noise and human performance by means of a subsidiary task measure. Fournal of Applied Psychology 54, 3 (1970), 211.

[5] Sandra R Harris, Robert L Kemmerling, and Max M North. 2002. Brief virtual reality therapy for public speaking anxiety. Cyberpsychology \& behavior 5, 6 (2002), 543-550. 
[6] HELENA HONG. 2010. TO ERR IS HUMAN: THE EFFECTS OF DISTRACTION ON ERRORS IN SKILLED PERFORMANCE. Ph.D. Dissertation. School of Psychology, University of New South Wales.

[7] Tomoko Kishimoto and Xinfang Ding. 2019. The influences of virtual social feedback on social anxiety disorders. Behavioural and cognitive psychotherapy 47, 6 (2019), 726-735.

[8] Merel Krijn, Paul MG Emmelkamp, Roeline Biemond, Claudius de Wilde de Ligny, Martijn J Schuemie, and Charles APG van der Mast. 2004. Treatment of acrophobia in virtual reality: The role of immersion and presence. Behaviour research and therapy 42, 2 (2004), 229-239.

[9] Merel Krijn, Paul MG Emmelkamp, Ragnar P Olafsson, and Roeline Biemond. 2004. Virtual reality exposure therapy of anxiety disorders: A review. Clinical psychology review 24, 3 (2004), 259-281.

[10] Joung Huem Kwon, John Powell, and Alan Chalmers. 2013. How level of realism influences anxiety in virtual reality environments for a job interview. International journal of human-computer studies 71, 10 (2013), 978-987.

[11] Pontus Larsson, Aleksander Väljamäe, Daniel Västfjäll, Ana Tajadura-Jiménez, and Mendel Kleiner. 2010. Auditory-induced presence in mixed reality environments and related technology. In The engineering of mixed reality systems. Springer, 143-163.

[12] Kwan Min Lee. 2004. Presence, explicated. Communication theory 14, 1 (2004) $27-50$.

[13] Russell A McCann, Christina M Armstrong, Nancy A Skopp, Amanda EdwardsStewart, Derek J Smolenski, Jennifer D June, Melinda Metzger-Abamukong, and Greg M Reger. 2014. Virtual reality exposure therapy for the treatment of anxiety disorders: an evaluation of research quality. Journal of anxiety disorders 28,6 (2014), 625-631.

[14] Fariba Mostajeran, Melik Berk Balci, Frank Steinicke, Simone Kühn, and Jürgen Gallinat. 2020. The Effects of Virtual Audience Size on Social Anxiety during Public Speaking. In 2020 IEEE Conference on Virtual Reality and 3D User Interfaces (VR). IEEE, 303-312

[15] Dinesh Nagar and Janak Pandey. 1987. Affect and Performance on Cognitive Task as a Function of Crowding and Noise. Journal of Applied Social Psychology 17, 2 (1987), 147-157.

[16] Max M North, Sarah M North, and Joseph R Coble. 1998. Virtual reality therapy: an effective treatment for the fear of public speaking. International fournal of
Virtual Reality 3, 3 (1998), 1-6.

[17] James C Overholser. 2002. Cognitive-behavioral treatment of social phobia. Journal of Contemporary Psychotherapy 32, 2-3 (2002), 125-144

[18] Thomas D Parsons and Albert A Rizzo. 2008. Affective outcomes of virtual reality exposure therapy for anxiety and specific phobias: A meta-analysis. Fournal of behavior therapy and experimental psychiatry 39, 3 (2008), 250-261.

[19] Gordon L Paul. 1966. Insight vs. desensitization in psychotherapy: An experiment in anxiety reduction. Stanford University Press.

[20] David-Paul Pertaub, Mel Slater, and Chris Barker. 2002. An experiment on public speaking anxiety in response to three different types of virtual audience. Presence: Teleoperators \& Virtual Environments 11, 1 (2002), 68-78.

[21] Ayelet Meron Ruscio, Timothy A Brown, Wai Tat Chiu, Jitender Sareen, Murray B Stein, and Ronald C Kessler. 2008. Social fears and social phobia in the USA: results from the National Comorbidity Survey Replication. Psychological medicine 38, 1 (2008), 15-28.

[22] Elham Salehi, Mahdi Mehrabi, Farhad Fatehi, and Asiyeh Salehi. 2020. Virtual Reality Therapy for Social Phobia: A Scoping Review. Studies in health technology and informatics 270 (06 2020), 713-717. https://doi.org/10.3233/SHTI200253

[23] G Serafin and S Serafin. 2004. Sound design to enhance presence in photorealistic virtual reality. Georgia Institute of Technology.

[24] Alexander Toet and Martin G van Schaik. 2012. Effects of signals of disorder on fear of crime in real and virtual environments. Fournal of Environmental Psychology 32, 3 (2012), 260-276.

[25] Paul Toprac and Ahmed Abdel-Meguid. 2011. Causing fear, suspense, and anxiety using sound design in computer games. In Game sound technology and player interaction: Concepts and developments. IGI Global, 176-191.

[26] F Vanni, C Conversano, A Del Debbio, Paola Landi, M Carlini, C Fanciullacci, and L Dell'Osso. 2013. A survey on virtual environment applications to fear of public speaking. European review for medical and pharmacological sciences 17, 12 (2013), 1561-1568.

[27] Isabelle Viaud-Delmon, Olivier Warusfel, Angeline Seguelas, Emmanuel Rio, and Roland Jouvent. 2006. High sensitivity to multisensory conflicts in agoraphobia exhibited by virtual reality. European Psychiatry 21, 7 (2006), 501-508.

[28] Bob G Witmer and Michael J Singer. 1998. Measuring presence in virtual environments: A presence questionnaire. Presence 7, 3 (1998), 225-240. 